\title{
Multistep radial melting in small two-dimensional classical clusters
}

\author{
D. M. Tomecka, ${ }^{*}$ B. Partoens, ${ }^{\dagger}$ and F. M. Peeters ${ }^{*}$ \\ Department of Physics, University of Antwerp (CMI), Groenenborgerlaan 171, 2020 Antwerp, Belgium
}

(Received 16 March 2005; published 23 June 2005)

\begin{abstract}
We report on a molecular dynamics study of small classical two-dimensional clusters with ringlike configurations. We focus on the particles motion at low temperatures before the radial and angular melting sets in. It is shown that in magic number configurations a local radial melting of subshells occur, which is related to the intershell rotation.
\end{abstract}

DOI: 10.1103/PhysRevE.71.062401

PACS number(s): 82.70.Dd, 45.05.+x, 36.40.Ei, 83.10.Tv

\section{INTRODUCTION}

Charged particles in two-dimensional (2D) clusters which are confined by a parabolic potential order themselves in shells, if the number of particles is not too large $(N<50)$. A Mendeleev type of table was constructed in Ref. [1] for these "classical atoms." Often, such a shell has a fine structure, it does not correspond with a well-defined ring, but consists of a few subshells, i.e., the shell has a nonzero width. In this paper we consider the small temperature behavior of those small clusters with ringlike ground state configurations.

The melting of 2D finite size crystals of charged particles is well studied [1-4]. Clusters consisting of not too many particles $(N<50)$ do not have a sharply defined melting temperature and its melting proceeds in a two-step process [1]. Initially, there is an orientational disordering characterized by the relative rotation of neighboring shells in which the internal order is retained. With further increase in temperature, intershell diffusion starts and the system melts radially. In a recent study of the melting properties of a Coulomb bound cluster it was also found that a structural phase transition can occur before the system melts [5].

In this paper, we found that the melting process of $2 \mathrm{D}$ classical clusters is much richer than represented by the above two-step approach. Here, we present a detailed investigation of the particles motion of small clusters as a function of temperature before any jumps between shells occur and thus before the radial melting sets in. We found that the detailed behavior depends very crucially on the exact ground state configuration and that temperature induced structural transitions are possible which lead to an enhanced symmetry with increasing temperature.

This paper is organized as follows. In Sec. II the model system and our numerical approach are introduced. Section III discusses the results and in Sec. IV our conclusions are summarized.

\section{MODEL SYSTEM AND NUMERICAL APPROACH}

We study the time dependence of a $2 \mathrm{D}$ system consisting of $N$ charged particles confined by a parabolic potential and

\footnotetext{
*Present address: Adam Mickiewicz University, Faculty of Physics, Umultowska 85, 61-614 Poznań, Poland.

${ }^{\dagger}$ Electronic address: bart.partoens@ua.ac.be

‡Electronic address: francois.peeters@ua.ac.be
}

interacting through a Coulomb interaction using molecular dynamics simulations. Using dimensionless units, the system of Newton equations which governs the time evolution of the $N$ particles in the cluster reads

$$
\frac{\partial^{2} \mathbf{r}_{i}}{\partial t^{2}}=-\frac{U\left(\mathbf{r}_{1}, \ldots, \mathbf{r}_{N}\right)}{\partial \mathbf{r}_{i}}, \quad i=1, \ldots, N,
$$

with

$$
U\left(\mathbf{r}_{1}, \ldots, \mathbf{r}_{N}\right)=\sum_{i=1}^{N} r_{i}^{2}+\sum_{i=1}^{N-1} \sum_{j=i+1}^{N} \frac{1}{\left|\mathbf{r}_{i}-\mathbf{r}_{j}\right|}
$$

the potential energy. Here, we used the following units for the time $t^{\prime}=\sqrt{2} / \omega_{0}$, frequency $\omega^{\prime}=\omega_{0} / \sqrt{2}$, length $r^{\prime}$ $=\left(2 e^{2} / m \epsilon \omega_{0}^{2}\right)^{1 / 3}$, energy $E^{\prime}=\left(m \omega_{0}^{2} e^{4} / 2 \epsilon^{2}\right)^{1 / 3}$, and temperature $T^{\prime}=E^{\prime} / k_{B}$, where $\omega_{0}$ is the confinement strength of the parabolic potential, $m$ is the mass of a particle and $e$ its charge, and $\epsilon$ is the static dielectric constant of the medium the particles are moving in.

As the start configuration for the molecular dynamics simulations the ground state configuration which minimizes the potential energy of Eq. (2) was used. It was obtained through a Monte Carlo simulation as explained in Ref. [2]. The set of Newton equations was solved using the velocity Verlet algorithm (with a typical stepsize of $\Delta t=10^{-2}$ which was tested to be sufficient to reach convergence). After rescaling the velocities to obtain the desired temperature, each simulation at a particular temperature consisted of $10^{3}$ equilibration time steps, followed by typically $10^{5}$ to $10^{6}$ time steps during which data was collected.

In order to study the dynamics of the particles, we calculated the mean square deviations. To characterize the radial melting processes we calculate the radial displacements,

$$
\left\langle u_{R}^{2}\right\rangle \equiv \frac{1}{N_{s}} \sum_{i=1}^{N_{s}}\left(\left\langle r_{i}^{2}\right\rangle-\left\langle r_{i}\right\rangle^{2}\right) / a^{2}
$$

where we introduced the averaged distance between the electrons $a=2 R_{\max } / \sqrt{N}$ with $R_{\text {max }}$ the maximal radius of the system. $\left\langle u_{R}^{2}\right\rangle$ is calculated for each shell separately and $N_{s}$ is the number of particles in each shell.

The angular melting is investigated using two quantities. The angular order in each shell is characterized by the angular intrashell displacements, 


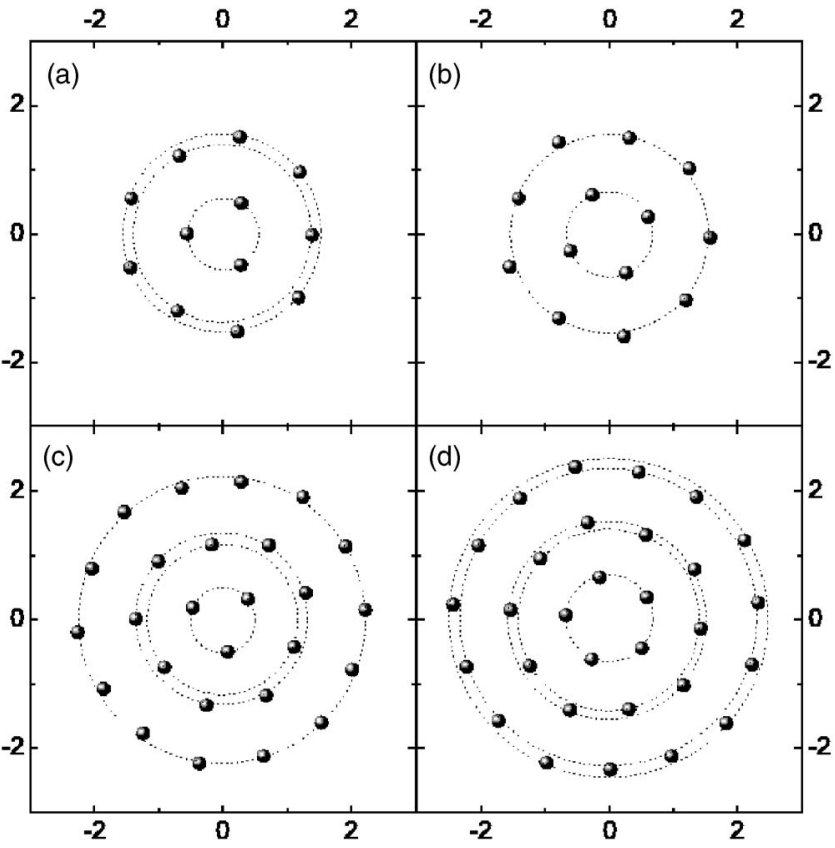

FIG. 1. The ground state configurations for (a) 12, (b) 13, (c) 26, and (d) 30 particles.

$$
\left\langle u_{\alpha_{1}}^{2}\right\rangle \equiv \frac{1}{N_{s}} \sum_{i=1}^{N_{s}}\left(\left\langle\left(\varphi_{i}-\varphi_{i_{1}}\right)^{2}\right\rangle-\left\langle\varphi_{i}-\varphi_{i_{1}}\right\rangle^{2}\right) /\left(\varphi_{0}^{(s)}\right)^{2},
$$

where $i_{1}$ indicates the nearest neighbor in the same shell and $\varphi_{0}=2 \pi / N_{s}$. The angular order between shells is characterized by the angular intershell displacements

$$
\left\langle u_{\alpha_{2}}^{2}\right\rangle \equiv \frac{1}{N_{s}} \sum_{i=1}^{N_{s}}\left(\left\langle\left(\varphi_{i}-\varphi_{i_{2}}\right)^{2}\right\rangle-\left\langle\varphi_{i}-\varphi_{i_{2}}\right\rangle^{2}\right) /\left(\varphi_{0}^{(s)}\right)^{2},
$$

where $i_{2}$ indicates the nearest particle from a neighboring shell. The division in shells and the determination of the nearest neighbors is made at the beginning of each temperature run.

Because of the small system size of the studied clusters there is no definite melting temperature but rather a melting region. Nevertheless we can define critical temperatures via

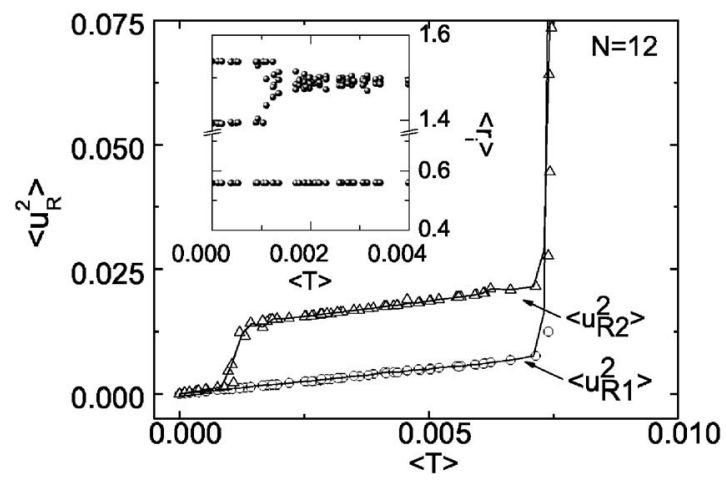

FIG. 2. The radial displacements for the inner and outer shell of the system of 12 particles as a function of the temperature. The inset shows the average radial position of each particle.

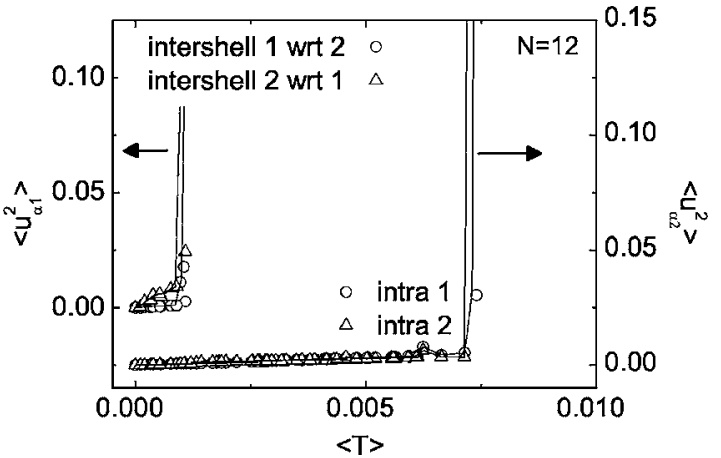

FIG. 3. The relative inter- and intrashell displacements for the same system with 12 particles as considered in Fig. 2. Left scale: the relative intershell displacements for shell 2 with respect to shell 1 , and shell 1 with respect to shell 2 as function of temperature. Right scale: the relative angular intrashell for both shells as a function of temperature.

the Lindemann criterion for melting. This implies that melting occurs when one of the above quantities $\left\langle u^{2}\right\rangle$ approaches 0.1 [6] or 0.05 for each coordinate.

\section{RESULTS}

As an example we considered a system containing 12 particles at low temperatures. The ground state corresponds to an inner ring with three particles and an outer ring with nine particles, indicated as $(3,9)$, which is shown in Fig. 1(a). This is a magic configuration [2], namely it is very stable against perturbations. The outer shell can be divided into two subshells, the outer subshell of the outer shell consists of six particles and the inner subshell of the outer shell consists of three particles, each having a slightly different radius, as indicated by the two rings in the outer shell in Fig. 1(a). Figure 2 shows the radial displacements for both shells as a function of the temperature. From the Lindemann criterion one finds that the radial melting for both shells sets in around $T_{r}$ $=0.0075$. Note that there is a remarkable jump in the radial displacement of the outer ring around $T=0.001$. This jump was never noticed before, probably because one needs rather accurate simulations for many temperature steps leading to long calculation times.

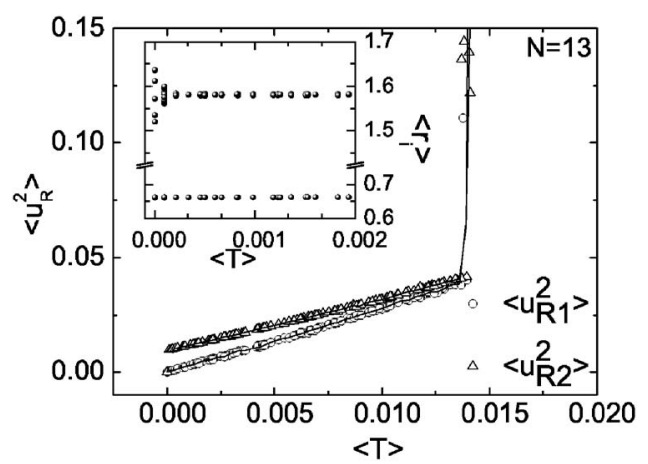

FIG. 4. The radial displacements for the inner and outer shell of the system of 13 particles as a function of temperature. The inset shows the average position of each particle. 


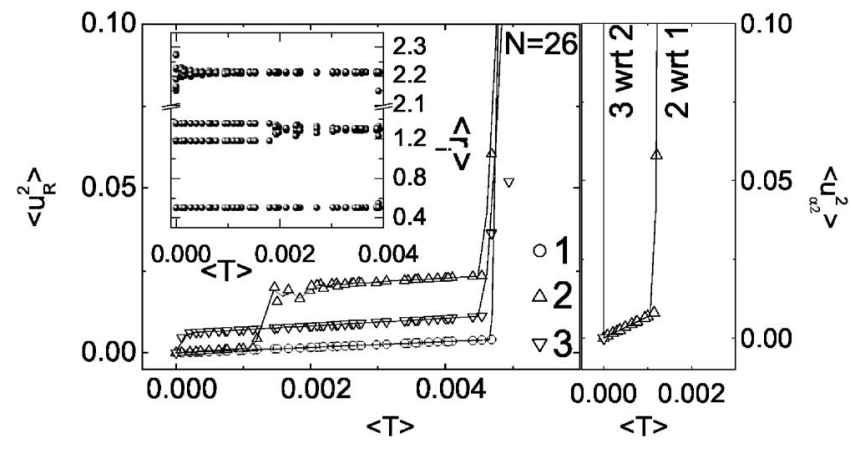

FIG. 5. (a) The radial displacements and the average positions (inset) of the particles for $N=26$ as a function of temperature. The numbers correspond to the number of the shell. (b) The corresponding relative intershell deviations for shell 3 with respect to shell 2 and shell 2 with respect to shell 1 as a function of temperature.

This jump can be understood by investigating the average position of each particle as a function of temperature. These results are shown in the inset of Fig. 2, where at zero temperature the three-ring structure is clearly seen with the outer ring showing a fine structure resulting in two subshells. However, exactly at the temperature where the jump in the mean square radial deviation occurs for the outer ring, the distinction between both outer subshells disappears and they coalesce in a single ring with the radius between the radius of the previous two subshells. We can conclude that this jump corresponds to the local melting of the outer rings, forming a single broader ring. This leads to an increased symmetry of the system. Consequently, the transition is a temperature induced structural (phase) transition. In this respect, it is similar to the structural phase transition which was recently found for certain Coulomb bound clusters [5].

At this jump, the angular order within each ring is not yet destroyed, as shown in Fig. 3, which shows the relative angular intrashell displacements of both shells. The relative intershell displacement (also shown in Fig. 3) on the other hand, is lost at exactly the same temperature where this radial melting of the outer ring sets in.

As the system of 12 particles is a magic configuration, it is interesting to see how the above results change for a nonmagic configuration. The ground state configuration for the system with 13 particles is $(4,9)$ [see Fig. 1(b)]. The radial displacement for both shells is shown in Fig. 4 and the average position of the particles in its inset. The outer ring can now be divided up in three subshells at zero temperature. With the slightest increase of temperature, they form one broad ring. This local radial melting goes hand in hand with the loss of intrashell angular order.

Finally, to show that this local radial melting is a general property of ringlike configurations, we consider the configurations with 26 and 30 particles. The ground state for 26 particles is $(3,9,19)$ [see Fig. 1(c)]. The first and second (inner) shells form a commensurate configuration, while the second and third (outer) shells are noncommensurate. The ground state for 30 particles is a fully magic configuration, namely $(5,10,15)$ [see Fig. 1(d)]. From Fig. 5(a), which

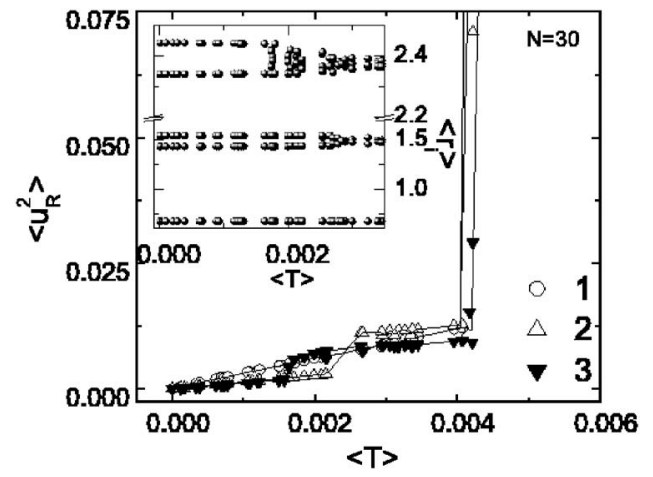

FIG. 6. The radial displacements for the three shells of the cluster consisting of 30 particles as a function of temperature. The inset shows the average position of each particle.

shows the radial displacements and the average positions (inset of Fig. 5), one can see that the subshells of the outer shell melt at very low temperature, while the radial order in the second subshell is kept until $T \approx 0.0015$. At this temperature, the orientational order between the first and second shell is also lost, as shown in Fig. 5(b), which shows the relative intershell deviations. This cluster exhibits both melting behaviors found in the previous clusters with $N=12$ and 13. In the fully magic configuration of 30 particles, a step occurs in the radial displacements of shell 2 at $T \approx 0.0025$ and shell 3 at $T \approx 0.0016$, as shown in Fig. 6 . The radial order is maintained longer in the second shell as it forms a magic configuration with the inner as well as with the outer ring.

\section{CONCLUSIONS}

The radial fluctuations of particles confined in small classical 2D clusters with ringlike configurations are investigated in the small temperature range before intershell diffusion sets in using molecular dynamics simulations. Some shells of the clusters have a fine structure and consist of a few subshells with slightly different radii. It was shown that the radial order in such a shell is maintained for magic configurations until the angular order between the different shells is lost. At this temperature, a jump in the mean square radial displacement is observed, and the different subshells form one broad ring, while the angular order in the shell is maintained. This corresponds with a local melting of the shell resulting in larger radial fluctuations. The other shells are not involved in this process and stay ordered. After this transition the symmetry of the cluster is increased and the particles in the broadened shell can interchange their positions.

In nonmagic configurations, the radial order in a shell is lost immediately after the temperature differs from zero, i.e., the smallest temperature fluctuations destroy the multisubshell order.

\section{ACKNOWLEDGMENTS}

This work was supported by the Flemish Science foundation (FWO-Vl.) and D.M.T. was supported by a EU Marie Curie training program. 
[1] V. M. Bedanov and F. M. Peeters, Phys. Rev. B 49, 2667 (1994).

[2] V. A. Schweigert and F. M. Peeters, Phys. Rev. B 51, 7700 (1995).

[3] Minghui Kong, B. Partoens and F. M. Peeters, Phys. Rev. E 67, 021608 (2003).

[4] R. Bubeck, C. Bechinger, S. Neser, and P. Leiderer, Phys. Rev.
Lett. 82, 3364 (1999).

[5] W. P. Ferreira, B. Partoens, F. M. Peeters, and G. A. Farias, Phys. Rev. E 71, 021501 (2005).

[6] Yu. E. Lozovik and V. M. Fartzdinov, Solid State Commun. 54, 725 (1985); V. M. Bedanov, G. V. Gadiyak and Yu. E. Lozovik, Phys. Lett. 109A, 289 (1985). 